\title{
Uso de ferramentas colaborativas por equipes em um ambiente acadêmico
}

\author{
Gennyson Filho, UNIFEI, gegecoucdout look . com, ORCID: 0000-0002-8909-3746 \\ Elisa de Cássia Silva Rodrigues, UNIFEI, elisa.rodrigues @unifei .edu.br, ORCID: 0000-0002-4649-1832 \\ Adriana Prest Mattedi, UNIFEI, amattedi@unifei . edu . br, ORCID:0000-0002-4605-9134
}

Resumo: Este artigo é um estudo de caso sobre como alunos na área de computação em uma Instituição de Ensino Superior (IES) percebem o uso de ferramentas colaborativas de desenvolvimento de software por equipes virtuais e híbridas. Utilizou-se o conceito de processos de equipe para definir os aspectos que compõem o trabalho em equipe e um questionário foi aplicado em três turmas na área de computação após a conclusão de um projeto nas disciplinas. A principal conclusão é que alguns sistemas colaborativos muito úteis na organização e execução dos projetos acabam falhando por não terem um canal de comunicação robusto que auxilie o gerenciamento da equipe e suas relações interpessoais, sendo necessário a utilização em conjunto com outras ferramentas.

Palavras-chave: sistemas colaborativos, processos de equipe, educação.

\section{Using Collaborative Tools by Teams in an Academic Environment}

Abstract: This article is a case study of how students in Computing Science courses at a Higher Education Institution (IES) perceive the use of collaborative tools for software development by virtual and hybrid teams. The concept of team processes was used to define the aspects that make up teamwork and a questionnaire was applied to three computer classes after the completion of a project in the courses. The main conclusion is that some collaborative systems that are especially useful for organizing and developing projects fail because they do not have a robust communication channel that helps the team management and its interpersonal relationships, requiring other support tools.

Keywords: collaborative systems, teams processes, education.

\section{Introdução}

O mundo globalizado, o desenvolvimento de novas Tecnologias de Informação e Comunicação (TICs), o aumento do nível educacional da população e, mais recentemente, a quarentena advinda da COVID-19 vêm afetando profundamente o ambiente educacional e as organizaç ões com relação a forma como se trabalha. Neste novo paradigma, o trabalho em equipe, a colaboração e a comunicação virtual se intensificaram tanto no ambiente acadêmico como no trabalho.

$\mathrm{O}$ conceito de equipe refere-se a dois ou mais indivíduos que estão interrelacionados para alcançar um objetivo em comum mas que, através de esforço coordenado, geram uma sinergia positiva (ROBBINS; JUDGE; SOBRAL, 2010). Entretanto, o resultado deste trabalho só é positivo se as atividades realizadas forem desenvolvidas em constante colaboração, sendo que o desempenho da equipe está positivamente relacionado a esta colaboração (FUKS; RAPOSO; GEROSA, 2003).

Com os avanços das redes e TICs a fim de facilitar essa nova dinâmica, baseada no empenho conjunto para realizar tarefas e projetos cada vez mais complexos e dispersos, uma gama de ferramentas virtuais tem sido desenvolvida, permitindo a criação de ambientes colaborativos com diversos fins (ANDRIESSEN, 2012). Um dos frutos dos avanços atuais é a computação em nuvem, que consiste em um modelo que disponibiliza acesso, conveniente e sob demanda, a diversos recursos computacionais configuráveis (servidores, aplicaç ões etc.), sendo providos fácil e rapidamente ao usuário, com esforço mínimo de gestão ou interação com um provedor de Internet (MILLER, 2008). 
Essa tecnologia tem sido usada por variadas organizações, inclusive as educacionais (ZUNGUZE; TSAMBE, 2020).

Neste contexto, surgiram os ambientes integrados de desenvolvimento ou IDEs (Integrated Development Environment) com o objetivo de oferecer vários recursos, localizados na nuvem, para o desenvolvimento de aplicações com colaboração em tempo real (ARORA; DIXIT, 2016). As IDEs online trazem uma nova dinâmica e desafios no relacionamento entre os membros das equipes. Por serem tecnologias emergentes, há a necessidade de se compreender qual o impacto destas no trabalho de uma equipe, em especial, na integração entre os membros e na execução de tarefas.

O objetivo deste trabalho é analisar, através de um estudo de caso, a percepção discente sobre o uso de ferramentas colaborativas para desenvolvimento de softwares em atividades de grupo no contexto acadêmico sob perspectiva dos processos de equipe. $\mathrm{O}$ estudo de caso aborda atividades de programação em contexto distintos, executadas por grupos de alunos do ensino superior que cursam computação, trabalhando de forma virtual ou híbrida. A pesquisa almeja adicionar ao cenário acadêmico uma nova abordagem que possa mapear aspectos relevantes a esse domínio.

Os referencial teórico sobre equipe e sistemas colaborativas são apresentados na Seção 2. A Seção 3 apresenta o método utilizado na realização da pesquisa. Na Seção 4, são analisados os resultados obtidos. Por fim, na Seção 5 são discutidas as conclusões da pesquisa.

\section{Referencial Teórico}

A sociedade sempre se organizou em equipes, seja por necessidade ou por identidade social. No decorrer dos anos, o papel das equipes foi se alterando para se adaptar a evolução da sociedade e das TICs e, com isso, a virtualização de equipes tem se mostrado cada vez mais importante em ambientes educacionais e organizacionais (KIRKMAN; GIBSON; KIM, 2012).

A literatura apresenta diversas classificaç ões para equipes que consideram a virtualização e o impacto causado por ela (KIRKMAN; MATHIEU, 2005; SCHWEITZER; DUXBURY, 2010; ROBBINS; JUDGE; SOBRAL, 2010). A classificação proposta por Robbins, Judge e Sobral (2010), por exemplo, subdivide as equipes em autogerenciadas, multifuncionais, de soluções de problemas e virtuais. Nesta classificação, equipe virtual pode ser definida como um conjunto de indivíduos que fazem parte de um mesmo grupo de trabalho com metas compartilhadas, mas que se encontram dispersos em lugares ou tempos diferentes, e se unem através das TICs para trabalhar.

Equipes virtuais vêm sendo utilizadas por organizaç ões desde a década de 90 a fim de viabilizar negócios descentralizados, processo este intensificado devido a vantagens como a redução de custos, globalização, flexibilidade e tecnologias disruptivas (HOLTON, 2001). Essa tendência vem ganhando espaço também nos ambientes educacionais com a Educação a Distância $(\mathrm{EaD})$ cada vez mais presente na vida dos acadêmicos. Em 2020, com o advento da quarentena imposta pela pandemia de COVID-19, o trabalho remoto e a educação a distância ganharam destaques sem precedentes em todo o mundo sendo, muitas vezes, a única opção segura para realização das atividades (ZUNGUZE; TSAMBE, 2020). Na atualidade, praticamente toda equipe realiza alguma parte do seu trabalho remotamente.

Devido a distância entre os membros e possíveis falhas de comunicação, uma equipe virtual pode encontrar dificuldades quanto à coordenação, confiança, motivação e comprometimento de seus membros (HUANG; KAHAI; JESTICE, 2010). Apesar de existirem dificuldades em equipes físicas também, alguns problemas como 
compartilhamento de informações de forma correta (ROBBINS; JUDGE; SOBRAL, 2010) ou sentimento de isolamento devido à falta de contato social direto acabam ficando mais evidentes em equipes virtuais.

As inovações das últimas décadas permitiram conciliar atividades em grupo com o uso do computador e possibilitaram a criação dos sistemas colaborativos ou groupware que pode ser definido como sistemas computacionais que oferecem a possibilidade de diversas pessoas se engajarem em uma mesma atividade, provendo um ambiente compartilhado para elas (ELLIS; GIBBS; REIN, 1991).

O modelo 3C - coordenação, cooperação e comunicação - é utilizado em sistemas colaborativos e visa explicar a relação cíclica que cada um desses elementos têm entre si para haver colaboração. As interações que ocorrem durante a atividade em grupo geram resultados que precisam ser administrados através da coordenação, que será responsável por definir as tarefas que serão executadas por cada um dos membros do grupo de forma cooperativa. Para cooperar será necessário ter comunicação entre os participantes fechando o ciclo da colaboração. Tais características estão em contato direto com a percepção, a qual é responsável por informar aos participantes sobre o que está ocorrendo ao seu redor, o que cada membro está fazendo e o que é possível absorver do ambiente para servir de auxílio na execução de alguma tarefa (FUKS; RAPOSO; GEROSA, 2003).

Estudos nas áreas de groupware e Trabalho Cooperativo Auxiliado por Computador (CSCW - Computer Supported Cooperative Work) deram origem a diversas classificações de sistemas colaborativos. A maioria dos autores se baseia no conceito de matriz de espaço/tempo, proposta por Johansen (1988), que divide os sistemas colaborativos de acordo com o tempo (tempo real ou tempo diferente) ou distância (mesmo lugar ou lugar diferente).

Alguns sistemas colaborativos podem se enquadrar em mais de um tipo de groupware devido a sua complexidade, como por exemplo, um sistema de gerenciamento de documentos compartilhados, em que é possível editar em tempo real ou diferente e que a funcionalidade de controle de versão pode ser usada em lugares dispersos ou no mesmo lugar. Penichet et al. (2007) apresentaram uma nova taxonomia que inclui as principais características de um CSCW, (compartilhamento de informação, comunicação e coordenação). Em seguida, Andriessen (2012) os dividiu entre sistemas de comunicação, compartilhamento de informações, cooperação, coordenação e encontros sociais, cada qual podendo também ser dividido em síncrono, assíncrono ou presencial.

A possibilidade de várias pessoas realizarem atividades de um mesmo projeto em tempo real foi o que motivou empresas a utilizarem o poder computacional dos clusters para oferecer ferramentas baseadas na web, inclusive colaborativas (MILLER, 2008). Inicialmente, esses sistemas eram usados apenas no meio empresarial, porém com o passar do tempo e a popularização da Internet, passaram a ser utilizados também por qualquer pessoa, em qualquer lugar. Exemplos dessas ferramentas vão desde ferramentas de e-mail até sistemas de edição de texto, com integração de chat, vídeo, controle de versão, entre outros. Posteriormente, pesquisas mostraram que muitas aplicações colaborativas baseadas na web facilitam o desenvolvimento do trabalho em equipe, habilidades sociais e computacionais (BOTTGE et al., 2009). Empresas de tecnologia, como a Google, disponibilizam serviços na nuvem que englobam diversas ferramentas colaborativas bem estabelecidas as quais são cada vez mais utilizadas no meio educacional e que possibilitam a execução de uma mesma tarefa por várias pessoas, reduzindo a necessidade por interações, bastando uma conexão com a Internet (OISHI, 2007).

Entre estudantes e profissionais de computaçãoé comum o uso de ferramentas para coordenação de projeto e de equipe como Trello (〈https://trello.com $\rangle$ ) e para 
hospedagem e controle de versão de código como GitHub ( $\langle$ https://github.com/ $\rangle$ ). Outra ferramenta para cooperação e compartilhamento de informações no contexto de programação são os ambientes integrados de desenvolvimento na nuvem, as cloud IDEs que se encontram em servidores online como o Codenvy ( $\langle$ https://codenvy.com/ $\rangle$ ). As cloud IDEs apresentam vantagens como redução de custos e de dificuldades com a manutenção de servidores e instalação de softwares locais, além de proporcionar um ambiente de programação centralizado para edição de um código de forma conjunta, permitindo assim a colaboração entre programadores localizados em lugares distintos, acesso fácil e rápido ao código e bibliotecas, acessibilidade em qualquer dispositivo reduzindo a duplicidade de trabalho (ARORA; DIXIT, 2016).

Ferramentas colaborativas são usadas principalmente em projetos cuja comunicação da equipe costuma ter um papel primordial. Atualmente, redes sociais como o Facebook e aplicativos de mensagens instantâneas como WhatsApp são bastante populares sendo usadas massivamente pela população em geral, inclusive no meio acadêmico, muitas vezes substituindo a tradicional comunicação por chamadas telefônicas (BOUHNIK; DESHEN, 2014).

Pesquisas nesta área vêm aumentando com o decorrer dos anos. $\mathrm{Na}$ área acadêmica Favela e Pena-Mora (2001) apontaram a dificuldade de integração online de alunos de engenharia de software que não se conheciam como obstáculo para o êxito dos projetos. Já Swigger et al. (2006) mostraram que a comunicação, infraestrutura e liderança são essenciais para o uso eficaz de sistemas colaborativos. Em um estudo sobre uma plataforma de desenvolvimento síncrona e colaborativa, Meneely e Williams (2009) mostraram que os alunos que a utilizaram tiveram resultados melhores do que aqueles que não o fizeram. Ainda, em 2014, Hidayanto e Setyady (2014) mostraram que a facilidade de uso e a utilidade percebida das ferramentas colaborativas levaram os alunos a usar as mesmas ao realizarem suas tarefas com um desempenho positivo da equipe.

Com a COVID-19, o uso de sistemas colaborativos se intensificou. Ao estudarem o Google Sala de Aula como recurso pedagógico durante o período da pandemia, Santos et al. (2021) inferiram que a falta de acesso à Internet e despreparo de alunos e professores quanto a sua utilização criaram dificuldade na sua utilização, mas que, por outro lado, criou dinamicidade e interatividade na sala de aula. Ainda durante a pandemia, Diaz e Rodero (2020) analisaram como os alunos de informática abordaram as tarefas de programação em grupo e os alunos adotaram um modelo de programação baseado na divisão do trabalho ou programação distribuída por pares, e muito poucos optaram por fazer uso de ferramentas de colaboração distribuída síncrona.

\section{Material e Métodos}

Para a realização da pesquisa, foram propostas atividades nas disciplinas Computação Gráfica, A lgoritmos e Estruturas d e D ados I e D esenvolvimento d e J ogos d os cursos Ciência da Computação (CCO) e Sistemas de Informação (SIN) da Universidade Federal de Itajubá. As tarefas consistiam no desenvolvimento de um projeto específico para cada disciplina que deveriam ser feitas em equipes. Os alunos poderiam escolher a(s) ferramenta(s) colaborativa(s) que julgassem mais apropriadas para a execução do projeto, coordenação e comunicação da equipe durante a realização do mesmo. No final da execução dos projetos, foi aplicado um questionário aos alunos de forma a obter a percepção dos mesmos sobre o uso destas ferramentas. O questionário foi respondido por 90 alunos, divididos em 28 equipes (de 2 a 5 alunos), e todos concordaram em participar voluntariamente da pesquisa. Cabe ressaltar que a pesquisa de campo foi realizada no ano de 2019, portanto, antes da pandemia de COVID-19. 
O questionário foi dividido em quatro partes. O primeiro bloco, com 12 questões, buscou informações sobre os participantes da pesquisa. As questões dos blocos 2, 3 e 4 foram baseadas na teoria de processos de equipe de Marks, Mathieu e Zaccaro (2001) e no método Net Promoter Score (NPS). Segundo Reichheld (2004), o NPS é um método de classificação da percepção de usuários sobre determinado produto ou serviço que, considerando uma escala de notas entre zero e 10, relaciona a reposta em promotores (notas 9 ou 10), passivos (notas 7 ou 8) ou detratores (notas de 0 a 6).

A teoria de Marks, Mathieu e Zaccaro (2001) aponta que os processos de uma equipe podem ser subdivididos em processos de transição (planejamento do projeto), ação (execução do projeto) e interpessoais, como mostra a Figura 1.

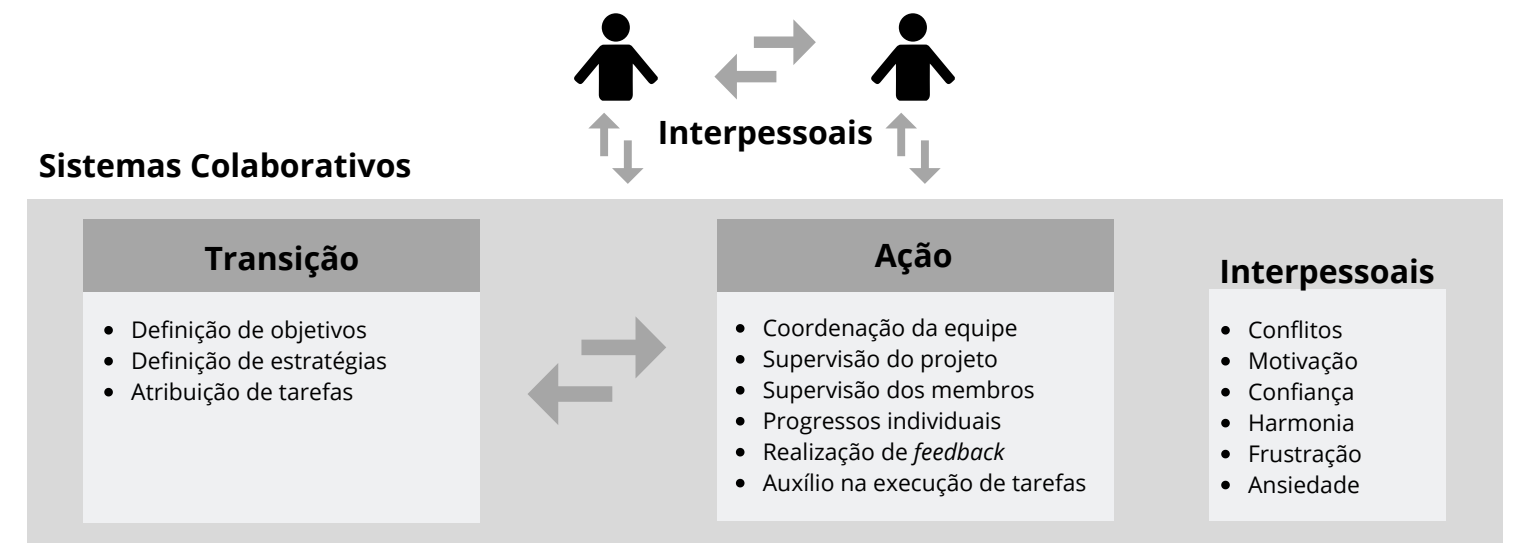

Figura 1. Tópicos relacionados aos processos de equipe (transição, ação e interpessoais) e relação destes em ambientes colaborativos. Fonte: Autoria própria.

Os processos de transição e de ação possuem uma relação cíclica, isto é, mudanças em um processo podem acarretar em ajustes de processos da outra fase. Já os processos interpessoais apoiam-se nas relações que norteiam o comportamento dos membros da equipe e permeiam ambos os processos de transição e de ação. Nas relações interpessoais, é comum o desenvolvimento de conflitos, confiança, afeto, coesão e integração social (ROBBINS; JUDGE; SOBRAL, 2010). Assim, nos blocos 2, 3 e 4 do questionário, os voluntários responderam quatro perguntas sobre cada um dos tópicos referentes aos processos de equipe (veja Figura 1): transição (12 questões), ação (24 questões) e interpessoais (24 questões). As quatro perguntas envolveram: qual a ferramenta mais utilizada; o quanto esta ferramenta auxiliou (usou-se o método NPS); se houve dificuldade com a ferramenta; e se houve, qual foi.

O questionário foi disponibilizado por meio do Google Forms, ferramenta online de formulários, e foi respondido pelos estudantes através da Internet em cerca de 15 minutos. Ressalta-se, ainda, que foi realizado um pré-teste do questionário com discentes de outra disciplina na mesma IES, com a finalidade de validar o questionário antes de ser aplicado na pesquisa em questão.

\section{Resultados e Discussões}

A Figura 2 apresenta um resumo das respostas em porcentagem que foram obtidas no bloco de perguntas preliminares do questionário. A maioria dos alunos respondeu que gosta de trabalhar virtualmente e em equipe, mas também indicaram pouca familiaridade entre os membros dos grupos. Estes dois pontos apontam problemas iniciais de integração mas também disposição para adaptação e aproximação. Mesmo assim, é possível que ocorram algumas dificuldades como, por exemplo, isolamento, comunicação, confiança e comprometimento ou, até mesmo, dificuldade em compartilhar informaç ões se a 


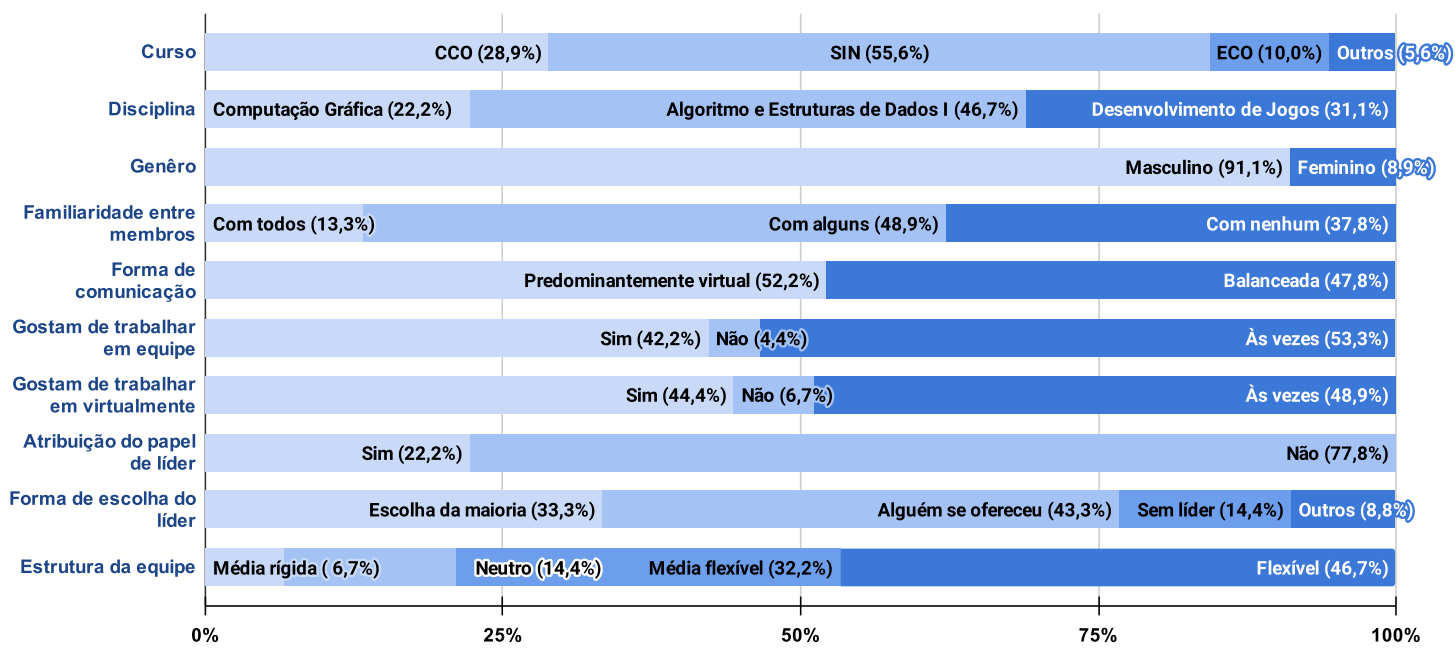

Figura 2. Respostas do bloco de perguntas preliminares. Fonte: Autoria própria.

integração não ocorrer, sendo a comunicação um fator decisivo. A forma de comunicação entre os membros das equipes não foi virtual a maior parte do tempo para a maioria das equipes, o que seria esperado na área de tecnologia de informação. Na realidade, apenas $52 \%$ dos respondentes afirmaram usar comunicação predominantemente virtual.

Embora o papel de líder tenha sido atribuído a algum membro da equipe (em geral, aquele que se ofereceu), a maioria deles informou que a estrutura da equipe foi flexível, o que implica em liderança compartilhada pelos membros. Destaca-se entre as vantagens de se ter um líder, o auxílio à equipe para solucionar problemas e influenciar positivamente o grupo a fim de alcançar os objetivos (ROBBINS; JUDGE; SOBRAL, 2010).

Os resultados sobre as ferramentas colaborativas mais escolhidas para cada tópico dos processos de equipe são apresentadas na Tabela 1 .

\begin{tabular}{lcccccc}
\multicolumn{6}{c}{ Tabela 1. Discentes que selecionaram a ferramenta por tópico dos processos de transição, ação e interpessoais (em \%) } \\
\hline Tópico dos processos & WhatsApp & Trello & Codenvy & GitHub & Google Docs & Facebook \\
\hline Definição de objetivos & 81,1 & 6,7 & 6,7 & - & - & - \\
Definição de estratégias & 74,4 & 6,7 & - & - & 6,7 & - \\
Atribuição de tarefas & 74,4 & 15,6 & - & - & - & 4,4 \\
\hline Coordenação da equipe & 77,8 & 10,0 & - & - & - & 4,4 \\
Supervisão do projeto & 55,6 & 12,2 & 14,4 & - & - & - \\
Supervisão dos membros & 56,7 & 16,7 & 10,0 & - & - & - \\
Progressos individuais & 82,2 & 4,4 & - & - & - & 4,4 \\
Realização de Feedback & 90,0 & 2,2 & 2,2 & - & - & 3,3 \\
Auxílio na execução de tarefas & 64,4 & - & 14,4 & 6,7 & - & - \\
\hline Gerenciamento de conflitos & 74,4 & - & - & - & - & - \\
Desenvolvimento de motivação & 80,0 & - & 4,4 & - & - & 5,6 \\
Desenvolvimento de confiança & 83,3 & - & 3,3 & - & - & 4,4 \\
Criação de harmonia & 85,6 & - & 3,3 & - & - & 5,6 \\
Gerenciamento de frustraçães & 90,0 & - & - & - & - & - \\
Gerenciamento de ansiedade & 73,3 & - & - & - & - & - \\
\hline
\end{tabular}

O primeiro bloco, processos de transição, envolveu a análise de três tópicos. Para definição de objetivos, o teste Kruskal-Wallis mostrou diferenças significativas ( $p=$ 0,008 ) nas percepç ões dos alunos sobre as três ferramentas mais escolhidas: o WhatsApp (65,8\% de promotores, $26 \%$ passivos e 8,2\% de detratores); o Trello apresentou 
mais neutralidade junto aos alunos (33,3\% de promotores, $50 \%$ passivos e $16,7 \%$ de detratores); e o Condenvy foi o que apresentou menor aceitação (16,7\% de promotores e 83,3\% de detratores). Já para o segundo tópico (definição de estratégias), o teste Kruskal-Wallis sobre as ferramentas mais escolhidas revelou diferenças significativas $(p=0,009)$ na visão dos alunos: WhatsApp com $62,7 \%$ promotores, $32,8 \%$ passivos e 4,5\% detratores; o Trello, neste quesito, apresentou mais detratores (50\%), 33,3\% promotores e 16,7\% passivos; e o Google Docs que apresentou uma neutralidade com $66,7 \%$ passivos e $33,3 \%$ promotores e $0 \%$ de detratores. Na avaliação dos alunos, ambas ferramentas em destaque para atribuição de tarefas, WhatsApp e Trello, foram bem aceitas com $64,2 \%$ de promotores enquanto opiniões passivas apresentaram valores de $28,4 \%$ e 21,5\%, respectivamente, e os detratores foram 7,5\% (WhatsApp) e 14,3\% (Trello).

Considerando que os processos de transição envolvem integração e forte comunicação da equipe, os resultados da pesquisa confirmam a expectativa de que um aplicativo de mensagens instantâneas, como o WhatsApp, seja a ferramenta mais eficiente para estes processos. O Trello também mostrou-se uma ferramenta importante aparecendo nas três etapas de transição, porém agradando mais na atribuição de tarefas, que é muito próprio dele. Por outro lado, ferramentas colaborativas boas para execução dos trabalhos compartilhados como o Codenvy e o Google Docs não agradaram os usuários no quesito comunicação tornando-se muitas vezes ineficientes para os processos de transição.

No segundo bloco foram analisados seis tópicos sobre os processos de ação. A Tabela 2 mostra a percepção dos respondentes sobre as ferramentas mais escolhidas para o desenvolvimento de cada um deles. Excetuando o tópico de feedback, a escolha das ferramentas foi mais diversificada nestes processos.

Tabela 2. Avaliação dos respondentes sobre as ferramentas escolhidas em cada item dos processos de ação (em \%)

\begin{tabular}{|c|c|c|c|c|c|c|c|}
\hline Ferramenta & $\begin{array}{c}\text { Tipo de } \\
\text { avaliação }\end{array}$ & $\begin{array}{c}\text { Coordenação } \\
\text { da equipe }\end{array}$ & $\begin{array}{c}\text { Supervisão } \\
\text { do projeto }\end{array}$ & $\begin{array}{c}\text { Supervisão } \\
\text { dos membros }\end{array}$ & $\begin{array}{l}\text { Progressos } \\
\text { individuais }\end{array}$ & Feedback & $\begin{array}{l}\text { Auxílio } \\
\text { em tarefas }\end{array}$ \\
\hline \multirow{3}{*}{ WhatsApp } & promotores & 61,4 & 56,0 & 58,8 & 66,2 & 65,4 & 56,9 \\
\hline & passivos & 32,9 & 32,0 & 23,5 & 24,3 & 25,9 & 29,3 \\
\hline & detratores & 5,7 & 12,0 & 17,6 & 9,5 & 8,6 & 13,8 \\
\hline \multirow{3}{*}{ Trello } & promotores & 66,7 & 54,5 & 53,3 & 75,0 & - & - \\
\hline & passivos & 33,3 & 18,2 & 26,7 & 25,0 & - & - \\
\hline & detratores & 0,0 & 27,3 & 20,0 & 0,0 & - & - \\
\hline \multirow{3}{*}{ Codenvy } & promotores & - & 30,8 & 22,2 & - & - & 23,1 \\
\hline & passivos & - & 38,5 & 33,3 & - & - & 23,1 \\
\hline & detratores & - & 30,8 & 44,4 & - & - & 53,8 \\
\hline \multirow{3}{*}{ GitHub } & promotores & - & 20,0 & 66,7 & - & - & 66,7 \\
\hline & passivos & - & 60,0 & 33,3 & - & - & 33,3 \\
\hline & detratores & - & 20,0 & 0,0 & - & - & 0,0 \\
\hline \multirow{3}{*}{ Facebook } & promotores & 25,0 & - & - & 25,0 & - & - \\
\hline & passivos & 25,0 & - & - & 50,0 & - & - \\
\hline & detratores & 50,0 & - & - & 25,0 & - & - \\
\hline
\end{tabular}

Os processos de ação têm uma característica um pouco distinta dos demais processos de equipe, sendo que a colaboração na execução do trabalho em si acaba se destacando igualmente, além da comunicação. Devido a isso, outras ferramentas como Trello e Codenvy também foram bastante escolhidas pelos discentes para estas tarefas, apesar de o WhatsApp ter se destacado novamente como a mais selecionada. Percebe-se que a seleção deste aplicativo de mensagens é recorrente pela familiaridade adquirida no seu rotineiro uso, mesmo não sendo o ideal para algumas tarefas. De acordo com a Tabela 2, o WhatsApp teve mais de 50\% de promotores em todos os tópicos. Entretanto, V.19 $\mathrm{N}^{\mathrm{o}} 1$, julho, 2021 
alguns respondentes relataram ter enfrentado dificuldades em usar tal aplicativo para o monitoramento do projeto devido ao excesso de informação dispersa sobre o projeto. A maioria dos alunos que escolheu o Codenvy também teve dificuldades com ela, deixando-a com alto número de detratores. Isso indica que o Codenvy precisa ser melhorado para se tornar uma ferramenta colaborativa que realmente facilite o trabalho virtual em grupo. O Trello também teve aprovação (mais de 50\% de promotores) nos quatro tópicos escolhidos pelos alunos, em especial nos tópicos monitoramento de trabalhos individuais (75\%) e coordenação de equipe $(66,7 \%)$.

Os processos interpessoais analisados envolvem seis tópicos (conflito, motivação, confiança, harmonia, frustração e ansiedade) e estão relacionados a como os membros da equipe interagem entre si. Ressalta-se que a boa condução destes elementos são a base para que os demais processos ocorram de forma eficiente. Assim como os processos de transição, os interpessoais também exigem bastante comunicação entre os membros do grupo. Portanto, novamente o WhatsApp foi destaque em todos os tópicos sendo que em três deles (conflitos, frustração e ansiedade) foi citada apenas esta ferramenta.

No item conflito, além do WhatsApp, 17,8\% dos respondentes apontaram que não houve este problema na equipe e, portanto, não necessitaram de ferramentas para lidar com isso. Outros 2,2\% disseram que a resolução seria melhor se feita pessoalmente, pois ocorria demora na resposta dos membros ou os membros não estavam dispostos a conversar sobre o conflito - o que piora se feito por aplicativos. Nos itens frustração e ansiedade, o WhatsApp teve aceitação média de 55\% e rejeição média de $16,4 \%$. Os respondentes apontaram que este tipo de aplicativo não é tão interessante para tratar estas questões uma vez que permite apenas informar sobre o que uma pessoa está sentindo, mas não permite um relacionamento mais humano que poderia melhorar a situação. A liderança também é um ponto importante nos processos interpessoais e uma das equipes apontou a falta dela como motivo do não incentivo para os membros comunicarem suas ansiedades durante o processo, mesmo elas existindo. Além disso, 20\% dos respondentes apontaram que não tiveram problemas de ansiedade nos seus grupos.

Nos itens motivação, confiança e harmonia, outras ferramentas além do WhatsApp foram apontadas pelos alunos (Codenvy e Facebook), mas que não tiveram boa aceitação. O WhatsApp apresentou cerca de $60 \%$ de promotores contra $11 \%$ de rejeição. Os principais pontos relatados foi que é difícil perceber o quão motivado um integrante está apenas com mensagens de texto, assim como manter a harmonia e confiança quando não se pode perceber o progresso feito. O Codenvy teve médias de 43,3\% de promotores e $35 \%$ de detratores e o Facebook teve uma aprovação média nestes itens de $19,4 \%$ e um índice médio de detratores de 41,7\%. Houve indiferença para com a ferramenta Facebook quando o foco era comunicação. Dessa forma, tal equipe parece ter encontrado na comunicação pessoal e nos laços de amizade uma forma de contornar os problemas causados pelas ferramentas utilizadas durante o processo de desenvolvimento de confiança. As equipes podem encontrar dificuldades na comunicação virtual, ainda mais se as ferramentas usadas carecem de recursos comunicacionais adequados (HUANG; KAHAI; JESTICE, 2010). Por fim, também ficou claro que há alunos que não gostam de usar ferramentas virtuais para se comunicar e dão preferência à comunicação presencial.

\section{Conclusões}

Neste trabalho, buscou-se investigar a percepção dos alunos da área de computação sobre o uso de ferramentas colaborativas no desenvolvimento de software por equipes virtuais/híbridas, as quais têm se tornado cada vez mais frequentes no ambiente V. $19 \mathrm{~N}^{\mathrm{o}} 1$, julho, 2021

DOI: https://doi.org/10.22456/1679-1916.118388 RENOTE 
organizacional pós COVID-19, sendo essencial que alunos sejam inseridos neste cenário.

Constatou-se que as ferramentas colaborativas analisadas possuem algumas facilidades percebidas pelos alunos como gerenciamento de arquivos e programação compartilhada em tempo real tornando a codificação e $\mathrm{m} g$ rupo m ais $\mathrm{f}$ ácil. Entretanto, também foi constatado que o desenvolvimento destas ferramentas nem sempre dá a importância devida para o item comunicação. Percebeu-se que a falta das funcionalidades adequadas gerou insatisfação e desistência do uso de algumas ferramentas analisadas, e apontou para a necessidade de um desenvolvimento melhor para que elas possam atingir a finalidade a qual s e p ropõem. Ferramentas d e m ensagens instantâneas s upriram em parte esta necessidade, mas também não foi considerada adequada em todo o processo, pois não é própria para a execução dos projetos e mesmo para resolução de problemas interpessoais.

Conclui-se então que uma ferramenta colaborativa deve ter funcionalidades robustas e bem definidas, facilidade de u so e fornecer u m bom c anal de comunicação, de forma que a coordenação do projeto seja eficaz. O múltiplo acesso, e m tempo real, de um documento único facilita a gestão do trabalho em equipe, desde que seja possível monitorar a atribuição de cada elemento, trazendo agilidade na execução do projeto.

Dado que a pesquisa foi feita antes da pandemia de COVID-19, sugere-se, como trabalho futuro, a análise de outras ferramentas colaborativas que foram desenvolvidas ou atualizadas com base nas necessidades do ensino e trabalho remoto durante a pandemia como, por exemplo, a cloud IDE Repl.it. Outra linha de pesquisa indicada é a investigação sobre ferramentas de comunicação como Google Meet e Zoom, utilizadas em atividades colaborativas, já que o uso destas se intensificou durante a pandemia.

\section{Referências}

ANDRIESSEN, J. H. E. Working with groupware: understanding and evaluating collaboration technology. [S.1.]: Springer Science \& Business Media, 2012.

ARORA, P.; DIXIT, A. Analysis of cloud ides for software development. International Journal of Engineering Research and General Science, v. 4, 2016.

BOTTGE, B. A.; RUEDA, E.; KWON, J. M.; GRANT, T.; LAROQUE, P. Assessing and tracking students' problem solving performances in anchored learning environments. Educational Tech Research and Development, Springer, v. 57, n. 4, p. 529-552, 2009.

BOUHNIK, D.; DESHEN, M. WhatsApp Goes to School: Mobile Instant Messaging between Teachers and Students. Journal of Information Technology Education: Research, v. 13, p. 217-231, 2014.

DIAZ, A.; RODERO, C. L. Group programming in COVID-19 time: The experience of the students of the ESI-CR of the UCLM. In: . [S.1.]: CEUR-WS.org, 2020.

ELLIS, C. A.; GIBBS, S. J.; REIN, G. Groupware: Some Issues and Experiences. Commun. ACM, ACM, NY, USA, v. 34, n. 1, p. 39-58, 1991. ISSN 0001-0782.

FAVELA, J.; PENA-MORA, F. An experience in collaborative software engineering education. Software, IEEE, v. 18, p. 47 - 53, 042001.

FUKS, H.; RAPOSO, A.; GEROSA, M. A. Do Modelo de Colaboração 3C à Engenharia de Groupware. In: WebMedia. [S.1.: s.n.], 2003. p. 0-8.

HIDAYANTO, A.; SETYADY, S. Impact of collaborative tools utilization on group performance in university students. Turkish Online Journal of Educational 
Technology, Sakarya University, v. 13, n. 2, p. 88-98, jan. 2014. ISSN 1303-6521.

HOLTON, J. Building trust and collaboration in a virtual team. Team Performance Management, v. 7, p. 36-47, 2001.

HUANG, R.; KAHAI, S.; JESTICE, R. The Contingent Effects of Leadership on Team Collaboration in Virtual Teams. Computers in Human Behavior, Elsevier, Amsterdam, Netherlands, v. 26, n. 5, p. 1098-1110, 2010. ISSN 0747-5632.

JOHANSEN, R. GroupWare: Computer Support for Business Teams. New York, NY, USA: The Free Press, 1988. ISBN 0029164915.

KIRKMAN, B.; GIBSON, C.; KIM, K. Across borders and technologies: Advancements in virtual teams research. Oxford Handbook of Organizational Psychology, v. 2, 2012.

KIRKMAN, B. L.; MATHIEU, J. E. The dimensions and antecedents of team virtuality. Journal of management, Sage Publications Sage CA, v. 31, n. 5, p. 700-718, 2005.

MARKS, M.; MATHIEU, J.; ZACCARO, S. A Temporally Based Framework and Taxonomy of Team Processes. Academy of Management Review, v. 26, p. 356, 2001.

MENEELY, A.; WILLIAMS, L. On preparing students for distributed software development with a synchronous, collaborative development platform. SIGCSE Bull., ACM, New York, NY, USA, v. 41, n. 1, p. 529-533, mar. 2009. ISSN 0097-8418.

MILLER, M. Cloud Computing: Web-Based Applications That Change the Way You Work and Collaborate Online. 1. ed. [S.1.]: Publishing Company, 2008.

OISHI, L. Working Together: Google Apps Goes to School. Technology \& Learning, ERIC, v. 27, n. 9, p. 46, 2007.

PENICHET, V.; MARIN, I.; GALLUD, J. A.; LOZANO, M.; TESORIERO, R. A classification method for C SCW s ystems. Electronic Notes in Theoretical Computer Science, v. 168, p. 237-247, 2007.

REICHHELD, F. The One Number you Need to Grow. Harvard business review, v. 81, p. 46-54, 2004.

ROBBINS, S.; JUDGE, T.; SOBRAL, F. Comportamento organizacional: teoria e prática no contexto brasileiro. [S.1.]: Pearson Prentice Hall, 2010.

SANTOS, M.; JúNIOR, A.; MACHADO, L.; BILESSIMO, S. Possibilidades e dificuldades n a u tilização d o g oogle s ala de a ula: u m e studo d e c aso e $\mathrm{m}$ u ma escola pública brasileira. RENOTE, v. 18, p. 49-58, 012021.

SCHWEITZER, L.; DUXBURY, L. Conceptualizing and measuring the virtuality of teams. Inf. Systems Journal, Wiley Online Library, v. 20, n. 3, p. 267-295, 2010.

SWIGGER, K.; BRAZILE, R.; HARRINGTON, B.; PENG, X.; ALPASLAN, F. Teaching students how to work in global software development environments. In: 2006 Int Conference Collaborative Computing: Networking, Applications and Worksharing. [S.1.: s.n.], 2006. p. 1-7.

ZUNGUZE, M.; TSAMBE, M. Perception of Teachers in the use of Electronic Platforms to Support Face-to-Face Teaching During the term of The State of Emergency due to Covid-19: Case of the Pedagogical University of Maputo. RENOTE, v. 18, n. 2, p. 40-48, 2020. 